\title{
36-Month Follow-Up Study of Post-Intervention Chronic Heart Failure Patients
}

\author{
Haruka Otsu ${ }^{*}$, Michiko Moriyama ${ }^{2}$ \\ ${ }^{1}$ Graduate School of Health Sciences, Hirosaki University, Hirosaki City, Japan \\ ${ }^{2}$ Division of Nursing Science, Institute of Biomedical \& Health Sciences, Hiroshima University, Hiroshima City, \\ Japan \\ Email: $\underline{\text { h }}$ otsu@cc.hirosaki-u.ac.jp
}

Received 14 January 2014; revised 20 February 2014; accepted 28 February 2014

Copyright (C) 2014 by authors and Scientific Research Publishing Inc.

This work is licensed under the Creative Commons Attribution International License (CC BY). http://creativecommons.org/licenses/by/4.0/

(c) (i) Open Access

\begin{abstract}
Aim: Although numerous studies of disease management and case management of chronic heart failure (CHF) have been carried out, length of effectiveness after program commencement has not been examined, so we examined a follow-up study at 36 months after program commencement. Methods: Participants went for follow-up visits to one Japanese clinic which specializes in internal cardiovascular medicine and they were given diagnoses of CHF. 104 outpatients participated in this study and randomized control trial was implemented. An educational program was implemented for 6 months. The data were collected at baseline, 3, 6, 9, 12 months from both intervention and control groups and at 24 and 36 months from the intervention group. Results: There was significant improvement in New York Heart Association (NYHA) in the intervention group between baseline and 36 months. Improvement in weight monitoring and activities or exercise in the intervention group continued up to 36 months. Meanwhile, sodium restricted diets and quitting smoking and/or drinking depended on individual preference and it was difficult to make improvements in these areas. Conclusions: The educational program showed promise in preventing CHF outpatients from deteriorating significantly on a long-term basis as self-monitoring of activity and weight continued significantly and there were no participants with CHF who deteriorated in the intervention group at 36 months after program commencement, although the program aimed only to provide illness and self-management knowledge. On the other hand, future work will need to compare participants in this program to a control group over an extended period of time with consideration for relieving the burden of the control group.
\end{abstract}

\section{Keywords}

Chronic Heart Failure; Disease Management; Educational Program; Follow-Up Study;

\footnotetext{
${ }^{*}$ Corresponding author.
} 


\section{Self-Management}

\section{Introduction}

Chronic heart failure (CHF) patients whose data were: Brain Natriuretic Peptide (BNP) $\geq 500$ pg/ml, New York Heart Association (NYHA) $\geq 3$ and who had a history of hospital admission for heart failure showed higher mortality [1]. Therefore, it is very important for patients to prevent CHF deterioration and readmission to hospital. To that end, numerous studies of disease management and CHF case management programs were conducted [2]-[10].

Although numerous studies of disease management and CHF case management programs have been carried out, only a few studies examined the long-term effects of disease management and case management programs [6] [11]. Medical factors such as ischemic heart disease and abnormal cardiac rhythm were pointed out as factors in the deterioration of CHF [12]. On the other hand, excess intake of sodium and fluid due to deficiencies in self-management also related to deterioration factors in CHF [12] [13]. Hence, it was considered that deficiencies in implementation of self-management caused CHF deterioration and hospital readmission.

Otsu and Moriyama [11] did a 24-month long-term follow-up study after starting their disease management program which was the longest follow-up period among previous studies and the results confirmed that performance of self-management of activities and self-monitoring of weight was continuous and no participants deteriorated. Consequently, long-lasting effects were proven in the 24 months after program commencement in [11]. However, it is necessary to research further long-term effects of disease management programs for CHF because it has not yet been revealed how long effects will last. The purpose of this study is to conduct follow-up investigations of these effects up to 36 months after program commencement in Otsu and Moriyama [9].

\section{Method}

\subsection{Research Design}

One hundred thirty-two outpatients with CHF agreed to enroll in this study but 28 outpatients refused to participate when it started giving a total of 104 outpatients at baseline. A random number generated by a computer program and was used to assign participants to participate in the educational program or to receive standardized treatment. A randomized control trial was implemented with both the intervention and control groups from baseline to 12 months after the start of the program, and before/after the trial was used within the intervention group at 24 and 36 months.

\subsection{Timeframe}

Enrollment and a randomized controlled trial were conducted from August 20 to September 31, 2007. The first follow-up period 7 to 12 months after the educational program started was from March 20 to October 31, 2008 [9]. The second follow-up research 24 months after the program started was conducted from August 20 to October 31, 2009 [11]. The third follow-up research 36 months after the program started was conducted from August 20 to October 31, 2010.

Intervention and first follow-up period data collection was implemented in both the intervention and the control groups from baseline to 12 months. In addition, follow-up period data collection, which included the additional second and third follow-up period, was implemented only in the intervention group from baseline to 36 months because approximately $80 \%$ of the control group dropped out during the second and third period.

\subsection{Description of Participants}

Participants went for follow-up visits to a Japanese clinic that specialized in cardiovascular internal medicine and were diagnosed with CHF. Participants whose condition was either stage 2 or 3 in NYHA and who had the physical and cognitive abilities to practice the program were selected regardless of age, sex, basic disease, complicating diseases, medical treatment, type of participants' residence, and family composition.

The sample size was estimated at 100 participants as $\alpha, \beta$, and standard effect sizes for two-way analysis of 
variance were each $0.05,0.20$, and 0.70 , respectively. During the intervention period that was up until 6 months after the program started, 49 participants in the intervention group and 47 participants in the control group who completed the program were analyzed. During the first follow-up period, 7 to 12 months after program commencement, 47 participants in the intervention group and 47 participants in the control group were analyzed. During the second follow-up period, 13 to 24 months after program commencement, 41 participants in the intervention group were analyzed. During the third follow-up period, 25 to 36 months after program commencement, 36 participants in the intervention group were analyzed. On the other hand, approximately $80 \%$ of the control group dropped out during the second and third period, and were subsequently removed from analysis as shown in Figure 1. Therefore, in this follow-up study we used time series design regarding the intervention group.

There were 61 male (63.5\%) and 35 female (36.5\%) participants during the first follow-up period. Their average age was $73.1 \pm 8.8$ years. Participants aged 65 and over accounted for $81.4 \%$ of participants as a whole. There were 13 participants who lived alone (13.5\%) and 19 participants with a job (19.8\%). There were no significant differences between intervention and control groups regarding all data in a baseline as shown in Table 1.

There were 26 male (63.4\%) and 15 female (36.6\%) participants during the second follow-up period. Their average age was $74.0 \pm 9.4$ years. There were 5 participants who lived alone (12.2\%) and 9 participants with a job $(22.0 \%)$.

There were 23 male (63.9\%) and 13 female (36.1\%) participants during the third follow-up period. Their average age was $72.9 \pm 9.8$ years. There were 5 participants who lived alone (13.9\%) and 8 participants with a job (22.2\%).

\subsection{Research Method}

Brief overview of Otsu and Moriyama CHF disease management program [9].

The period of the educational program was set for 6 months because a minimum of 6 months was required for patients to acquire self-management skills [14] and the program was carried out for 30 minutes once a month individually with a total 6 sessions conducted with a pamphlet developed on the basis of evidence-based clinical guidelines [9]. After that, follow-up, which consisted of confirmation by interview of whether the intervention group continued to implement self-management and discussion of directions to achieve personal goal setting if their self-management was not continued appropriately, were conducted for an average of 20 minutes once a month for 6 months and the disease management program including a follow-up period which was carried out for a total of 12 months.

The 6 sessions consisted of an introduction to: How to adjust to CHF; Giving up smoking (if applicable); A letter to the family; A reduced sodium and fluids diet and giving up drinking; Self-management; Monitoring medicine administration; Activities and exercises; and How to control one's emotions. In particular, BNP, which was one of the deteriorative indicators of CHF, tended to get worse in the case of participants who consumed excess sodium [13], so the program placed an emphasis on sodium restriction.

The researcher provided knowledge backed up with pamphlets at each session and also gave guidance in accordance with the participants' actual life. In addition, goal setting for sodium intake was performed every month and the researcher had discussions with the each participant if they did not achieve their own goals. Regarding self-monitoring, a calendar was used to check compliance and weight. This aimed to prevent missed medication doses and to discover CHF deterioration at an early date such as potential edema through body weight gain. In this program, the degree of participants' heart function was mild to intermediate (New York Heart Association Class 2 to 3), so strict fluid restriction was discarded. It was excluded from goal setting even though knowledge on this subject was provided. The researcher and the participant confirmed and reviewed inspection data and compliance together at each visit and provided feedback for the participant and the primary doctor.

\subsection{Procedure and Period of Evaluation}

The program, developed by Otsu and Moriyama [9], was carried out for the intervention group. The control group received standardized treatment and care that was provided as general treatment and care according to the conditions of the individual at a medical facility. Self-monitoring implementation status in the intervention group was confirmed with individual interviews in the first follow-up period which was 6 months after imple- 


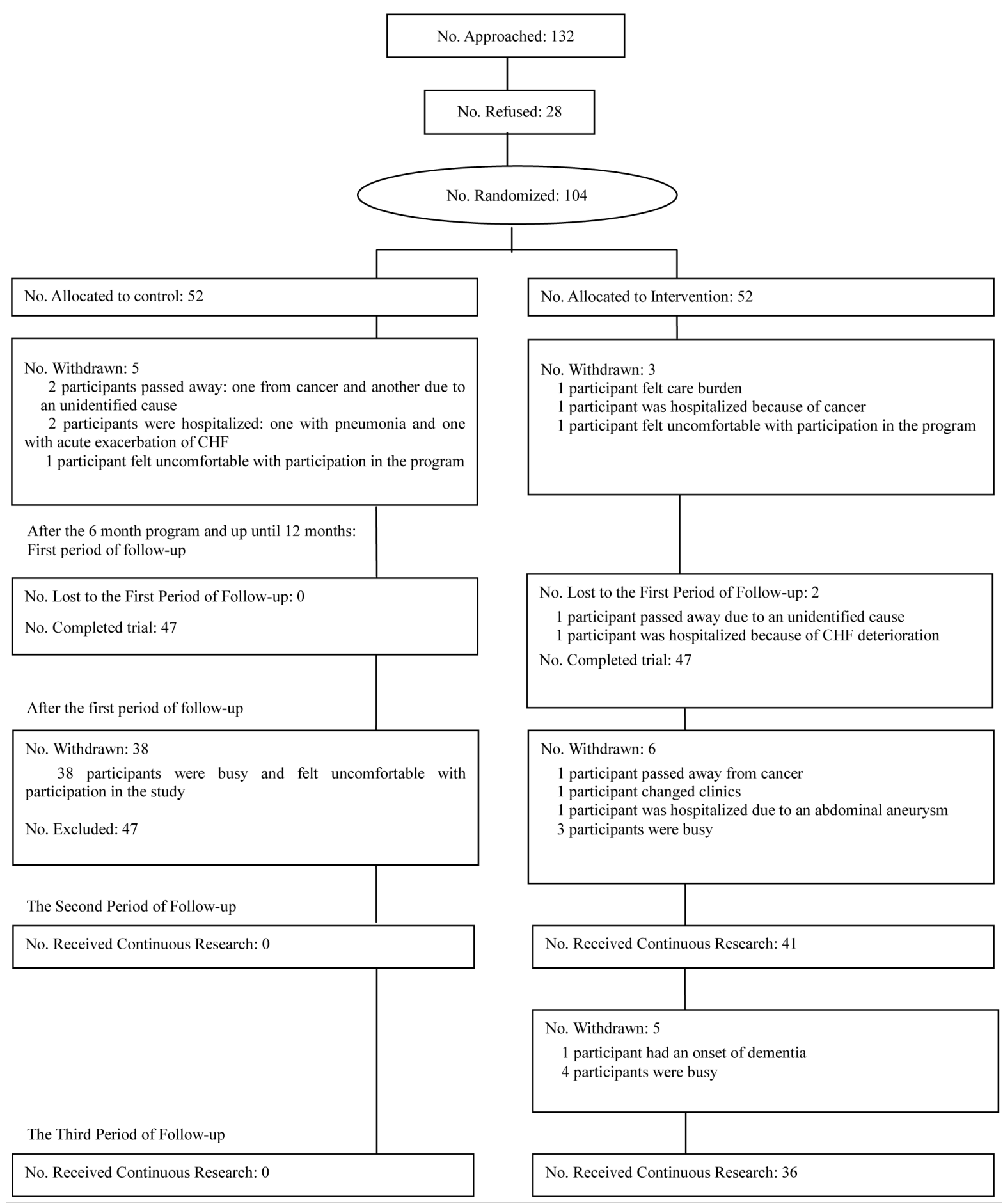

\section{Figure 1. Participant flow.}

mentation of the program. The individual interviews were conducted for an average of 20 minutes with self-reported measures to assess participants' compliance with a sodium-restricted diet, medicine administration, activities/exercises, weight-monitoring and giving up smoking and drinking. After that, confirmation with the second follow-up period continued for an additional 12 months after the first follow-up period. Furthermore, confirmation with the third follow-up period continued for an additional 12 months after the second follow-up period.

Evaluation was carried out at the time of enrollment (baseline), and at 6 months (completion of intervention), 
Table 1. Baseline comparison between groups.

\begin{tabular}{|c|c|c|c|}
\hline Demographic variable & Intervention group $(\mathrm{n}=49)$ & Control group $(n=47)$ & p-value \\
\hline \multicolumn{4}{|l|}{ Basic attribute } \\
\hline Male sex & $31(63.3 \%)$ & $30(63.8 \%)$ & 1.000 \\
\hline Age & $71.6 \pm 9.3$ & $74.6 \pm 8.1$ & 0.198 \\
\hline Living alone & $7(14.3 \%)$ & $6(12.8 \%)$ & 1.000 \\
\hline Worker & $13(26.5 \%)$ & $6(12.8 \%)$ & 0.125 \\
\hline \multicolumn{4}{|l|}{ Underlying disease } \\
\hline Hypertension & $40(81.6 \%)$ & $36(76.6 \%)$ & 0.620 \\
\hline Ischemic heart disease & $16(32.7 \%)$ & $22(46.8 \%)$ & 0.211 \\
\hline Valve disease & $24(49.0 \%)$ & $23(48.9 \%)$ & 1.000 \\
\hline Cardiac myopathy & $5(10.2 \%)$ & $1(2.1 \%)$ & 0.204 \\
\hline \multicolumn{4}{|l|}{ Blood pressure } \\
\hline Systolic pressure & $128.2 \pm 11.9$ & $131.2 \pm 7.0$ & 0.565 \\
\hline Diastolic pressure & $71.3 \pm 5.5$ & $71.5 \pm 4.7$ & 0.881 \\
\hline Pressure pulse & $57.7 \pm 10.0$ & $58.6 \pm 9.4$ & 0.656 \\
\hline Body weight & $62.1 \pm 11.4$ & $62.7 \pm 10.7$ & 0.780 \\
\hline \multicolumn{4}{|l|}{ Clinical condition } \\
\hline New York Heart Association grade 2 & 39 (79.6\%) & $38(80.9 \%)$ & 1.000 \\
\hline Brain natriuretic peptide & $153.2 \pm 149.1$ & $163.6 \pm 198.5$ & 0.769 \\
\hline Arrhythmia & $12(24.5 \%)$ & $11(23.4 \%)$ & 1.000 \\
\hline Paroxysmal dyspnea & $0(0 \%)$ & $0(0 \%)$ & - \\
\hline Distention of the jugular vein & $0(0 \%)$ & $0(0 \%)$ & - \\
\hline Moist rale & $0(0 \%)$ & $0(0 \%)$ & - \\
\hline Ankle edema & $3(6.1 \%)$ & $0(0 \%)$ & 0.242 \\
\hline Coughing at night & $0(0 \%)$ & $0(0 \%)$ & - \\
\hline Shortness of breath & $5(10.2 \%)$ & $6(12.8 \%)$ & 0.757 \\
\hline \multicolumn{4}{|l|}{ Curative drug } \\
\hline Acetylcholinesterase inhibitor & $5(10.2 \%)$ & $2(4.3 \%)$ & 0.436 \\
\hline$\beta$ blocker & $23(46.9 \%)$ & $17(36.2 \%)$ & 0.308 \\
\hline Digitalis product & $10(20.4 \%)$ & $13(27.7 \%))$ & 0.477 \\
\hline Diuretic & $24(49.0 \%)$ & $22(46.8 \%)$ & 0.841 \\
\hline Aldosterone antagonist & $18(36.7 \%)$ & $10(21.3 \%)$ & 0.118 \\
\hline Angiotensin-receptor antagonist & $16(32.7 \%)$ & $20(42.6 \%)$ & 0.400 \\
\hline
\end{tabular}

Otsu \& Moriyama, 2011 [9].

12 months (6 months after intervention), 24 months (18 months after intervention), and 36 months (30 months after intervention) after enrollment. Individual interviews were conducted with MacNew-QOL questionnaires whose validity and reliability were confirmed in Otsu et al. [15] and compliance to CHF self-management in a pre-determined format and clinical data were collected from medical reports. 


\section{Outcomes}

\subsection{Primary Outcomes}

Primary outcomes were identified as death due to CHF, hospital admission due to CHF, BNP data, NYHA level, blood pressure including systolic pressure, diastolic pressure, and pressure pulse, weight and deteriorating symptoms related to CHF such as arrhythmia, coughing at night, moist rale, ankle edema, and shortness of breath.

\subsection{Secondary Outcomes}

Secondary outcome were identified using the MacNew-QOL questionnaire, as developed by Höfer et al. [16] to measure the QOL of heart disease patients, including patients with coronary artery and/or heart failure. This original scale consisted of 27 items, from which we developed a Japanese version consisting of 19 items. Reliability, validity, and value were confirmed [15]. The subscale included emotional, physical, and social conditions with a seven grade scale. Scores were calculated from each condition or as a cumulative score, with a higher score representing a better condition.

\subsection{Process Indicators}

Process indicators were identified as: compliance to a sodium-restricted diet, compliance to medicine administration, compliance to activities or exercise, compliance to quit smoking, compliance to quit drinking, and selfmonitoring of weight and deteriorating symptoms. Compliance to sodium-restriction, medicine, activities or exercise, and self-monitoring weight were described on a 4-point scale (0 to 3), with a higher score representing greater compliance. Other indicators were evaluated as good or poor. The researcher assessed compliance on the basis of participants' self-reports.

\subsection{Method of Analysis}

Repeated two-way analysis of variance was performed on physiological, QOL, and compliance data [9]. The Wilcoxon rank-sum test was also conducted to compare time variations in each group to see if the results from repeated two-way analysis of variance showed significant difference in physiological, QOL, and compliance data. Regarding the compliance data in the process indicators, a four level score was given to each compliance performance: 3 was given to participants who were always in compliance; 2 was given to participants who were partially in compliance; 1 was given to participants who were slightly in compliance; and 0 was given to participants who were not in compliance at all. To make a distinction between partially and slightly compliant, the former meant that compliance was implemented more than 4 times per week and the latter meant that compliance was implemented less than 4 times per week in a pre-determined format. Regarding smoking and drinking, good in compliance meant that smoking and drinking were reduced to within 3 times per week and poor in compliance meant that smoking and drinking were continued more than 4 times per week. After that, the average score of compliance performance was calculated for each group. For categorical data, the chi-squared test was carried out. SPSS version 16.0 (SPSS, New York, New York, USA) was used to analyze and the level of significance was set at $p<0.05$.

\subsection{Ethical Considerations}

Approval from the ethical committee from the researchers' university was obtained and all necessary procedures for this research were carried out at the target facilities. The purpose and description of the study, the method and type of data collection, method of publication of the research results, voluntary participation, and refusal of participation that did not result in a disadvantage, and protection of privacy were all explained. Participant consent was obtained in writing.

\section{Results}

\subsection{Baseline Comparison between Groups}

Demographic variable in participants is shown in Table 1. There was no significant difference between two 
groups in baseline.

\subsection{Consideration of the Continuous Effectiveness of the Program}

Primary outcomes in intervention, the first, second, and third follow-up period.

The flow of participants in this study is shown in Figure 1. During the intervention period, one patient in the intervention group asked to withdraw from the study after one month. In the control group, five participants dropped out. During the first period of follow-up, from 7 to 12 months after the program started, in the intervention group, two participants dropped out. No participants in the control group dropped out during the first period of follow-up. In the intervention group, six participants dropped out during the second period of follow-up which was 13 to 24 months after the program started. Moreover, in the intervention group, five participants dropped out during the third period of follow-up which was 25 to 36 months after the program started. In the control group 38 participants dropped out during the second and third period, so all of the control group were excluded because of insufficient data.

There were no fatalities due to CHF in either group until the first period of follow-up 12 months after the start of the program. One participant in the control group was hospitalized due to CHF deterioration during the program. On the other hand, no one in the intervention group was hospitalized due to CHF deterioration during the program. One participant in the intervention group was hospitalized due to CHF deterioration for 30 days, six months later (after the end of program) and one participant from the control group was hospitalized for 90 days, three months after the program started. After that, no one was hospitalized due to CHF deterioration during the follow-up period up until 36 months.

There were no unscheduled clinic visits due to CHF deterioration up to 12 months in the intervention group. On the other hand, three participants made unscheduled visits to the clinic due to CHF deterioration in the control group, visiting 4, 7 and 25 times although the rate of scheduled clinic visits in each group varied depending on the participant such as once every two weeks, three weeks, or month. From 13 to 24 months, just one participant in the intervention group made unscheduled visits to the clinic, visiting 7 times. From 25 to 36 months, there were no unscheduled clinic visits due to CHF deterioration in the intervention group.

\subsection{Primary Outcomes of the Second and Third Follow-Up Period}

Physiological data are shown in Table 2. BNP data within the intervention group during the comparison period was significantly improved between baseline and 3 months $(p=0.032)$, and baseline and 6 months $(p=0.002)$ as a result of multiple comparisons. However, there was no significant difference regarding BNP in the intervention group in comparison between baseline and 36 months. On the other hand, participants in stage 3 of NYHA reduced to $8.3 \%$ in the intervention group after 36 months and significant differences were indicated between baseline and 36 months $(p<0.001)$ despite the fact that there were no significant differences regarding NYHA between intervention and control groups at 3, 6, 9, 12 months. Regarding weight, there were significant differences within the intervention group during the comparison period $(p<0.001)$ although there was no data in the before-after periods. Also, systolic blood pressure $(p=0.022)$ and pressure pulse $(p=0.033)$ were significantly improved within the intervention group during the comparison period, but there were no significant differences in the before-after periods.

There was no significant difference regarding shortness of breath in the intervention group through 36 months despite the fact that there were significant differences between intervention and control groups at 3, 9, 12 months (Table 3). Regarding coughing at night, no one in either group indicated the symptoms in any period and also there were no significant differences in either group in any period other than shortness of breath (Table 3).

\subsection{Secondary Outcomes in Intervention and the First, Second, and Third Follow-Up Period}

Results of the MacNew test are shown in Table 4. Interaction was shown in total, emotional, physical, and social scores between intervention and control groups as a result of repeated two-way analysis of variance. As a result of the Wilcoxon rank-sum test, total and emotional scores in the intervention group were significantly better between baseline and 6 months $(p<0.001)$, baseline and 12 months $(p<0.001), 6$ and 9 months $(p<$ 


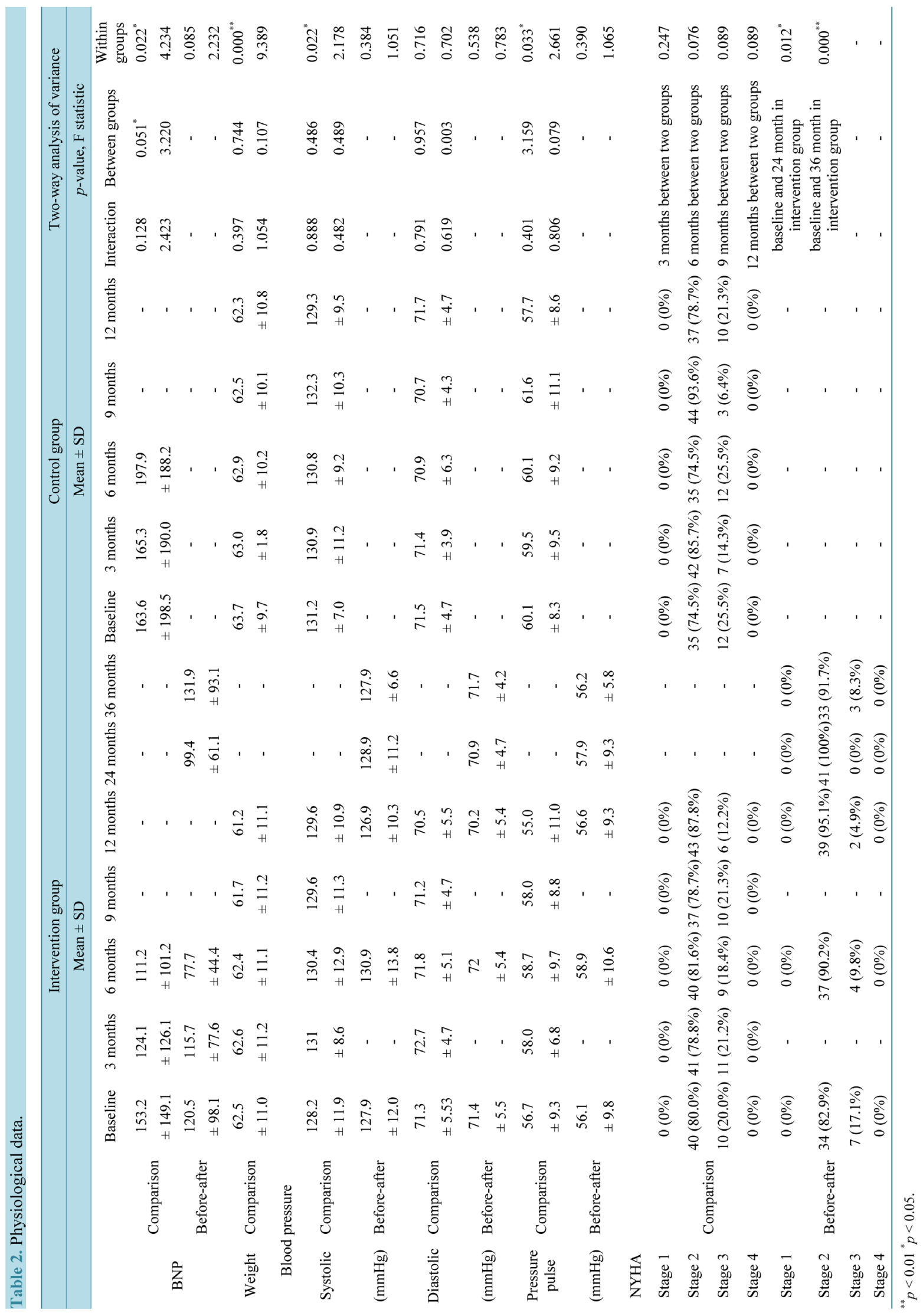


Table 3. Deteriorating symptoms related to CHF.

\begin{tabular}{|c|c|c|c|c|c|c|}
\hline & \multirow{2}{*}{ Period } & \multicolumn{2}{|c|}{ Intervention } & \multicolumn{2}{|c|}{ Control } & \multirow{2}{*}{ p-value } \\
\hline & & Existence & Non-existence & Existence & Non-existence & \\
\hline \multirow{7}{*}{ Arrhythmia } & Baseline & 12 (24.5\%) & 37 (75.5\%) & 11 (23.4\%) & $36(76.6 \%)$ & 1.000 \\
\hline & 3 months & 12 (24.5\%) & 37 (75.5\%) & 12 (25.5\%) & 35 (74.5\%) & 1.000 \\
\hline & 6 months & 11 (22.4\%) & 38 (77.6\%) & 12 (25.5\%) & 35 (74.5\%) & 0.813 \\
\hline & 9 months & 13 (26.5\%) & 36 (73.5\%) & 15 (31.9\%) & $32(68.1 \%)$ & 0.655 \\
\hline & 12 months & 13 (27.7\%) & 34 (72.3\%) & 13 (27.7\%) & 34 (72.3\%) & 1.000 \\
\hline & 24 months & $10(24.4 \%)$ & 31 (75.6\%) & - & - & - \\
\hline & 36 months & $9(25.0 \%)$ & 27 (75.0\%) & - & - & - \\
\hline \multirow{7}{*}{$\begin{array}{l}\text { Coughing at } \\
\text { night }\end{array}$} & Baseline & $0(0 \%)$ & 49 (100\%) & $0(0 \%)$ & 47 (100\%) & - \\
\hline & 3 months & $0(0 \%)$ & 49 (100\%) & $0(0 \%)$ & 47 (100\%) & - \\
\hline & 6 months & $0(0 \%)$ & 49 (100\%) & $0(0 \%)$ & 47 (100\%) & - \\
\hline & 9 months & $0(0 \%)$ & 49 (100\%) & $0(0 \%)$ & 47 (100\%) & - \\
\hline & 12 months & $0(0 \%)$ & $47(100 \%)$ & $0(0 \%)$ & $47(100 \%)$ & - \\
\hline & 24 months & $0(0 \%)$ & $41(100 \%)$ & - & - & - \\
\hline & 36 months & $0(0 \%)$ & $36(100 \%)$ & - & - & - \\
\hline \multirow{7}{*}{ Moist rale } & Baseline & $0(0 \%)$ & 49 (100\%) & $0(0 \%)$ & $47(100 \%)$ & - \\
\hline & 3 months & $0(0 \%)$ & 49 (100\%) & $0(0 \%)$ & 47 (100\%) & - \\
\hline & 6 months & $0(0 \%)$ & 49 (100\%) & $0(0 \%)$ & $47(100 \%)$ & - \\
\hline & 9 months & $1(2.0 \%)$ & 48 (98.0\%) & $1(2.1 \%)$ & 46 (97.9\%) & 1.000 \\
\hline & 12 months & $0(0 \%)$ & $47(100 \%)$ & $1(2.1 \%)$ & 46 (97.9\%) & 1.000 \\
\hline & 24 months & $1(2.4 \%)$ & 40 (97.6\%) & - & - & - \\
\hline & 36 months & $0(0 \%)$ & $36(100 \%)$ & - & - & - \\
\hline \multirow{7}{*}{ Ankle edema } & Baseline & 3 (6.1\%) & 46 (93.9\%) & $0(0 \%)$ & $47(100 \%)$ & 0.242 \\
\hline & 3 months & $5(10.2 \%)$ & 44 (89.8\%) & $0(0 \%)$ & 47 (100\%) & 0.056 \\
\hline & 6 months & $2(4.1 \%)$ & 47 (95.9\%) & $1(2.1 \%)$ & 46 (97.9\%) & 1.000 \\
\hline & 9 months & 2 (4.1\%) & 47 (95.9\%) & 2 (4.3\%) & 45 (95.7\%) & 1.000 \\
\hline & 12 months & $1(2.1 \%)$ & 46 (97.9\%) & $2(4.3 \%)$ & 45 (95.7\%) & 1.000 \\
\hline & 24 months & 2 (4.9\%) & 39 (95.1\%) & - & - & - \\
\hline & 36 months & $0(0 \%)$ & $36(100 \%)$ & - & - & - \\
\hline \multirow{7}{*}{$\begin{array}{l}\text { Shortness } \\
\text { of breath }\end{array}$} & Baseline & $5(10.2 \%)$ & 44 (89.8\%) & 7 (12.8\%) & $41(87.2 \%)$ & 0.757 \\
\hline & 3 months & 3 (6.1\%) & 46 (93.9\%) & 10 (21.3\%) & 37 (78.7\%) & $0.038^{*}$ \\
\hline & 6 months & 5 (10.2\%) & 44 (89.8\%) & 11 (23.4\%) & 36 (76.6\%) & 0.104 \\
\hline & 9 months & 3 (6.1\%) & 46 (93.9\%) & 12 (25.5\%) & 35 (74.5\%) & $0.011^{*}$ \\
\hline & 12 months & 3 (6.4\%) & 44 (93.6\%) & 11 (23.4\%) & $36(76.6 \%)$ & $0.041^{*}$ \\
\hline & 24 months & 3 (7.3\%) & 38 (92.7\%) & - & - & - \\
\hline & 36 months & 5 (13.9\%) & 31 (86.1\%) & - & - & - \\
\hline
\end{tabular}

${ }^{*} p<0.05$. 


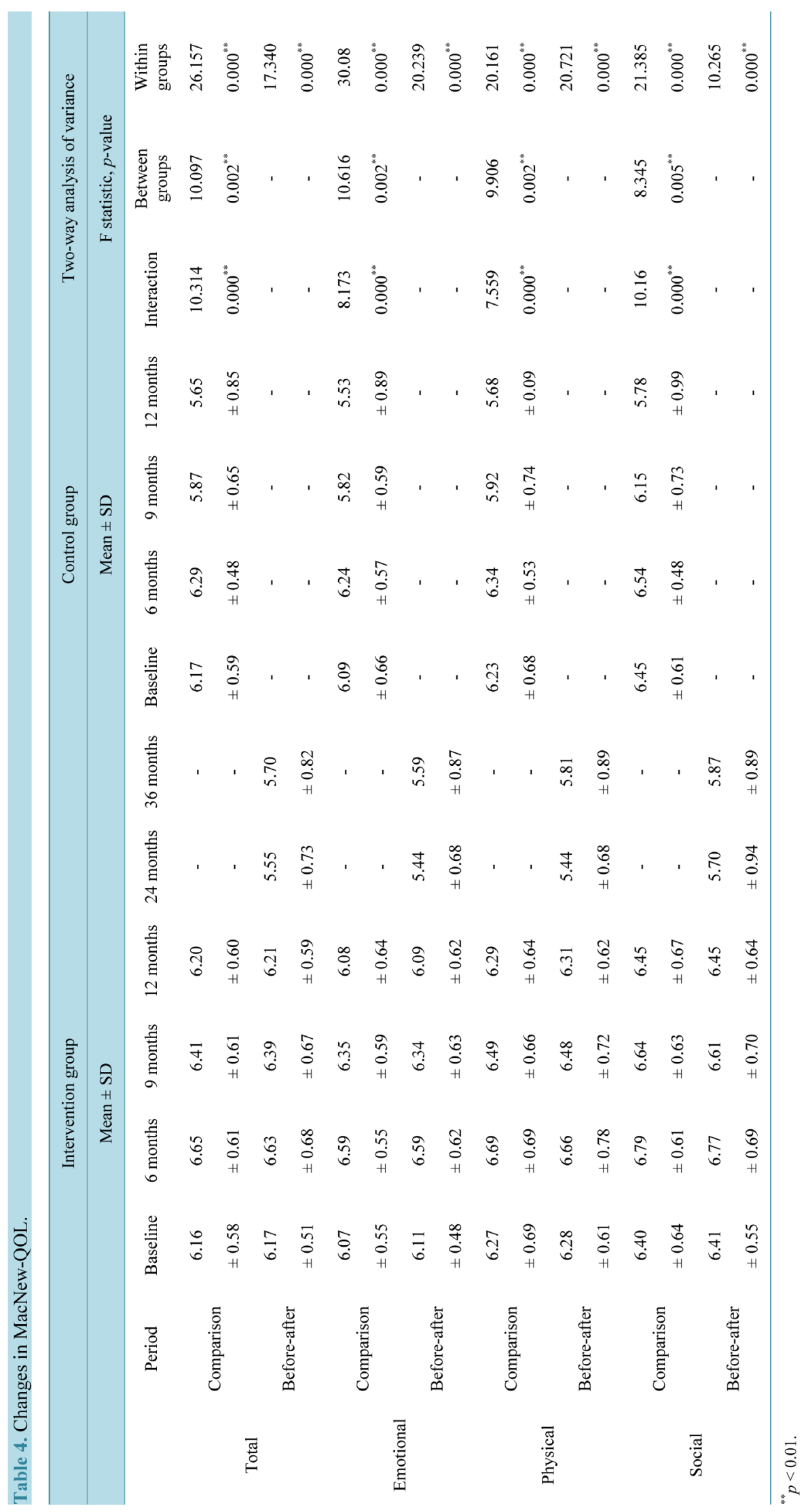


0.001), 6 and 12 months ( $p<0.001), 9$ and 12 months ( $p=0.002)$ in comparison with the control group. Also, a result of the Wilcoxon rank-sum test, physical scores in the intervention group were significantly better between baseline and 6 months $(p=0.002)$, baseline and 12 months $(p=0.001), 6$ and 9 months $(p<0.001), 6$ and 12 months $(p<0.001), 9$ and 12 months $(p=0.008)$ in comparison with the control group. Additionally, a result of the Wilcoxon rank-sum test, social scores in the intervention group were significantly better between baseline and 6 months $(p=0.001)$, baseline and 12 months ( $p<0.001), 6$ and 9 months $(p<0.001), 6$ and 12 months $(p<$ 0.001), 9 and 12 months $(p=0.001)$ in comparison with the control group. Briefly, QOL in the intervention group was better than the control group up to 12 months. After that, there were significant differences between baseline and 24 months regarding all scores, and there were significant differences between baseline and 36 months regarding total, emotional, and physical scores at a level of $1 \%$ in the intervention group during the second and third follow-up periods from 13 to 36 month as a result of the Wilcoxon rank-sum test. Basically, QOL achieved a peak in 6 months, became equal to baseline at 12 months, and finally after 24 and 36 months showed a decrease.

\subsection{Process Indicators in Intervention, the First, Second, and Third Follow-Up Period}

Implementation status of self-management is shown in Tables 5 and 6. Regarding the above-mentioned table, a four level score was given to each compliance performance: 3 was given to participants who were always in compliance; 2 was given to participants who were partially in compliance; 1 was given to participants who were slightly in compliance; and 0 was given to participants who were not in compliance at all. To make a distinction between partially and slightly compliant, the former meant that compliance was implemented more than 4 times per week and the latter meant that compliance was implemented less than 4 times per week. Regarding the latter table which was about smoking and drinking, good in compliance meant that smoking and drinking were reduced within 3 times per week and poor in compliance meant that smoking and drinking were continued more than 4 times per week. All of the data in the Tables 5 and 6 were obtained from self-reported data and were measured at a single point in time.

Interaction was shown in compliance with a sodium-restricted diet from baseline up to 12 months between intervention and control group as a result of repeated two-way analysis of variance. As a result of the Wilcoxon rank-sum test, compliance with a sodium-restricted diet in the intervention group was significantly better between baseline and 6 months ( $p<0.001)$, baseline and 9 months $(p<0.001)$, and baseline and 12 months $(p=$ 0.005 ) in comparison with the control group. On the other hand, compliance at 24 months and 36 months decreased compared to at 6 months in the intervention group. Similarly, compliance to medicine administration in the intervention group at 9 months was significantly high in comparison to baseline $(p<0.001)$, but compliance at 24 months and 36 months decreased compared to at 9 months as a result of the Wilcoxon rank-sum test.

Regarding weight monitoring, as a result of the Wilcoxon rank-sum test, weight-monitoring in the intervention group was significantly better between baseline and 6 months $(p<0.001)$, and baseline and 12 months $(p<$ $0.001)$ in comparison with the control group. Similarly, improvement continued until 36 months $(p<0.001)$ although compliance at $24(p=0.038)$ and 36 months $(p=0.023)$ decreased compared to at 9 months and compliance at 24 months $(p<0.001)$ decreased compared to at 6 months as a result of the Wilcoxon rank-sum test. Also, as a result of the Wilcoxon rank-sum test, compliance with activities/exercises in the intervention group was significantly better between baseline and 6 months $(p<0.001)$, baseline and 9 months $(p<0.001)$, baseline and 12 months $(p<0.001), 6$ and 9 months $(p=0.009)$, and 6 and $12(p=0.011)$ months in comparison with the control group. Similarly, good compliance with activities/exercise in the intervention group continued until 36 months $(p<0.001)$, although compliance in 24 months $(p=0.048)$ decreased compared to 9 months as a result of the Wilcoxon rank-sum test. However, there was no significant difference between the two groups up to 12 months regarding compliance with quitting smoking and drinking. When compared to baseline, compliance with quitting smoking continued for the most part, but compliance with stopping drinking was reduced at 24 and 36 months in the intervention group.

\section{Discussion}

\subsection{Improvement of Clinical Indicators and Effectiveness of the Intervention Program}

Regarding BNP data, there was no significant difference at 6, 12, 24, and 36 months after program commence- 


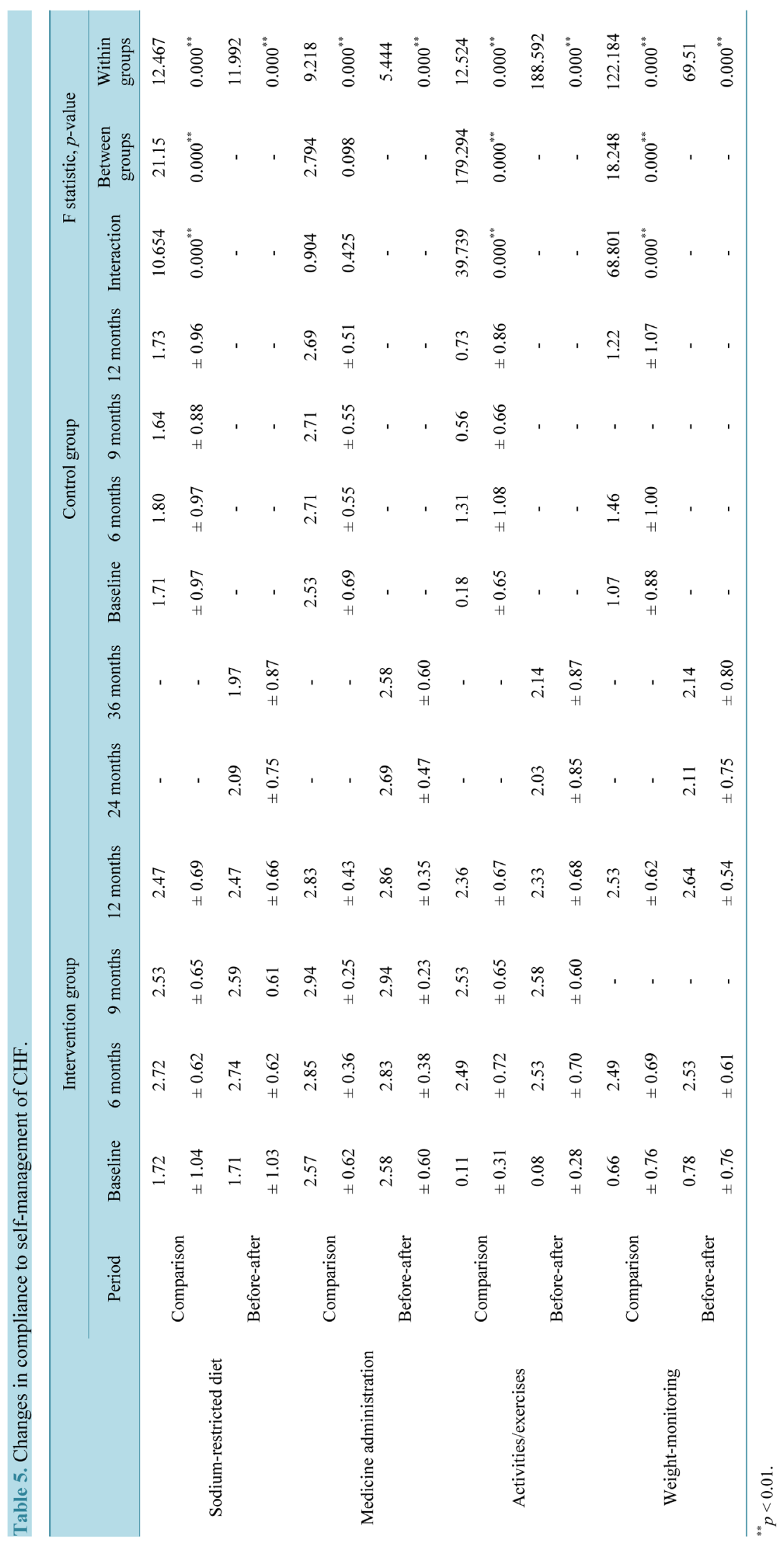


Table 6. Compliance with the self-management program for CHF.

\begin{tabular}{|c|c|c|c|c|c|c|}
\hline & \multirow{2}{*}{ Period } & \multicolumn{2}{|c|}{ Intervention } & \multicolumn{2}{|c|}{ Control } & \multirow{2}{*}{ p-value } \\
\hline & & Good & Poor & Good & Poor & \\
\hline \multirow{6}{*}{ Quite smoking } & Baseline & 42 (85.7\%) & 7 (14.3\%) & 46 (97.9\%) & $1(2.1 \%)$ & 0.059 \\
\hline & 6 month & 46 (93.9\%) & 3 (6.1\%) & 45 (95.7\%) & $2(4.3 \%)$ & 1.000 \\
\hline & 9 months & 44 (89.8\%) & 5 (10.2\%) & 46 (97.9\%) & $1(2.1 \%)$ & 0.204 \\
\hline & 12 months & 42 (89.4\%) & $5(10.6 \%)$ & 46 (97.9\%) & $1(2.1 \%)$ & 0.203 \\
\hline & 24 months & 36 (87.8\%) & $5(12.2 \%)$ & - & - & - \\
\hline & 36 months & 31 (86.1\%) & 5 (13.9\%) & - & - & - \\
\hline \multirow{6}{*}{ Quite drinking } & Baseline & 32 (65.3\%) & 17 (34.7\%) & 38 (80.9\%) & 9 (19.1\%) & 0.110 \\
\hline & 6 month & $28(57.1 \%)$ & $21(42.9 \%)$ & 32 (68.1\%) & 15 (31.9\%) & 0.298 \\
\hline & 9 months & $29(59.2 \%)$ & $20(40.8 \%)$ & 30 (63.8\%) & 17 (36.2\%) & 0.679 \\
\hline & 12 months & 27 (57.4\%) & 20 (42.6\%) & $28(59.6 \%)$ & 19 (40.4\%) & 1.000 \\
\hline & 24 months & $24(58.5 \%)$ & 17 (41.5\%) & - & - & - \\
\hline & 36 months & 20 (55.6\%) & $16(44.4 \%)$ & - & - & - \\
\hline
\end{tabular}

ment in the intervention group. However, BNP data in Otsu and Moriyama [9] was significantly improved at 6 months in the intervention group compared to the control group. Significant differences may be demonstrated if follow-up study between the intervention and control groups is implemented. On the other hand, NYHA levels were significantly improved between baseline and 24 months $(p=0.012)$ and baseline and 36 months $(p<0.001)$ in the intervention group. Additionally, deteriorating symptoms related to CHF were stable throughout the follow-up period, so it was considered that self-management behavior in the intervention group continued accordingly.

Regarding events caused by CHF deterioration until the first period of follow-up 12 months after the start of the program there were no participants who died due to CHF deterioration in either groups. However, one participant in each group was hospitalized due to CHF during the program, but the length of the hospitalization in the intervention group was 60 days shorter than the participant in the control group. In previous studies, death mortality or re-hospitalization due to CHF occurred in $64 \%$ of control patients and $47 \%$ of intervention patients in the 180-day follow-up period [17]. Similarly, death or re-hospitalization due to CHF occurred in 53\% of control patients and $37 \%$ of intervention patients at the one year mark [18]. In comparison to these previous studies, the ratio of death or re-hospitalization due to CHF in our study was quite low. The previous studies provided education just one time before discharge, and Koelling et al. [17] implemented follow-up by telephone at 30, 90, and 180 days after discharge, but there was no information about follow-up after discharge in Blue et al. [18]. On the other hand, our study provided 6 individual outpatient sessions and implemented face-to-face follow-up once a month for 12 months. Therefore, it is considered that targeted teaching to each participant and face-toface teaching methods for extended period of time may lead to building a relationship of trust between the researcher and the participants and to continued self-management by the participants. Additionally, 35 to 40 percent of severe CHF patients were included in previous studies, but we had no severe NYHA patients in either group. Thus, the status of heart function at baseline may relate to the low rate of death and hospitalization due to CHF in our study.

\subsection{Process Indicators in Intervention, the First, Second, and Third Follow-Up Period}

There is no significant difference in compliance with a sodium-restricted diet, medicine, giving up smoking or giving up drinking at 24 and 36 months after program commencement. On the other hand, compliance with activities/exercises and weight-monitoring were significantly improved between baseline and 24 months ( $p<$ $0.001)$ and baseline and 36 months $(p<0.001)$. As a result of basic research which aimed to develop the disease 
management program for CHF in Otsu and Moriyama [13], outpatients with CHF showed poor self-management because they had insufficient knowledge. To that end, the rate of execution of self-management improved after the program started because patients had knowledge of the illness and its self-management. Therefore, this was effective in compliance with activities/exercises and weight-monitoring although the program only provided knowledge to outpatients. On the other hand, a sodium-restricted diet or giving up smoking or drinking related to personal preference, so it was assumed that it was difficult for outpatients to continue adherence in selfmanagement behavior with a program that only provided knowledge. Activities/exercises were also related to personal preference, but good compliance with activities/exercise in the intervention group continued until 36 months. It was speculated that implementation of activities/exercise was not so difficult for the participants to continue because it was assigned just for 20 to 30 minutes three times a week in comparison to a sodium-restricted diet or giving up smoking or drinking which had to be accomplished at every meal or every day, although there are no previous studies to support this. Therefore, long-term follow-up was important for CHF outpatients so that they could continue adherence in self-management behavior for sodium-restricted diets and giving up smoking or drinking in particular. Motivational interviews should be used to continue participants' adherence in the long term because previous studies achieved an effect in patients' life style change with motivational interviews [19] [20].

The results of the study showed that good compliance with activities or exercise continued up to 36 months in the intervention group. However, there was no significant difference in compliance with a sodium-restricted diet, medicine and giving up smoking or drinking at 24 and 36 months after program commencement. According to guidelines for giving up smoking [21], smokers were addicted to nicotine, so it was difficult for them to give up smoking by sheer force of will on a long-term basis. Hence, medical treatment for giving up smoking was also needed for people whose degree of dependence was high. For the consumption of alcohol, according to evidence-based clinical guidelines about CHF [22], it was essential that people who had suspected alcoholic cardiomyopathy to give up drinking. On the other hand, for other people with cardiovascular disease, alcohol in moderation was recognized. However, in elderly patients with old myocardial infarcts, alcohol consumption and reinfarction were associated with sudden death [23], so it is important for patients with old myocardial infarcts to be heavily encouraged to give up. Moreover, compliance with a sodium-restricted diet was also an important factor because it related to deteriorating symptoms of CHF [13]. For the future, in addition to providing knowledge, it is important for patients with CHF to be educated about how to change their lifestyle and habits in order to maintain proper compliance.

\subsection{Secondary Outcomes}

Total, emotional, physical, and social scores in MacNew quality of life in the intervention group were significantly better at 6 months, but all scores were equal to baseline at 12 months, and furthermore all scores significantly decreased at 24 and 36 months compared to baseline. It was hard to compare this with a previous study which used the MacNew quality of life after a disease management program for CHF because very few studies used the MacNew quality of life scale. In other QOL scales, Stewart's study et al. [2] showed a nurse who carried out home visits for 7 to 14 days after the patients' hospital discharge and their QOL which was the Minnesota Living with Heart Failure Questionnaire was significantly improved at 3 months after intervention, but there was no significant difference at 6 months after intervention [2]. Also, West et al. [24] conducted home visits and follow-up by telephone once a week. After that, the QOL score which was SF36 was significantly improved at 6 months compared to baseline after intervention commencement [24]. However, over the following months, there was no follow-up data. In addition, in heart failure and/or cardiology outpatient clinics, patients with CHF participated in a six-week self-management program, and as a result, cognitive symptom management, self-care behavior, and QOL were significantly improved in Smeulders et al. [8]. They used the RAND 36-item Health Survey and the Kansas City Cardiomyopathy Questionnaire as the scale of QOL, and it was reported that there was no significant difference at 6 and 12 months in the follow-up period. Very few previous studies examined the long-term effects [11]. Hence, our study showed precious data because we collected long-term data up to 36 months after program commencement.

\subsection{Association with QOL and Aging}

In the study, at 12 months all scores for total, emotional, physical, and social categories in MacNew quality of 
life in the intervention group were equal to baseline and after that all scores significantly decreased at 24 and 36 months. One possible reason was that elderly participants were not inclined to go out or do anything due to their age. This became apparent in the individual data collection interviews at 24 and 36 months because 7 elderly participants answered as above. On the other hand, good compliance with activities or exercise continued up to 36 months in the intervention group. At the baseline, almost none of the participants knew how much activity or exercise was appropriate, so some of them tended to spend all their time indoors and others tended to move too much. As some participants got older, they tended to go out less. In addition to this, elderly participants with cataracts in this study felt boredom due to their advancing years because of alteration in visual acuity. It was considered that QOL scores for elderly participants decreased due to increasing age rather than the influence of CHF.

\subsection{Efficacy and Usability of this Study}

The disease management program in this study was effective in improving outcomes. Previous studies have also reported the effectiveness of the program [3] [17]. A disease management program implemented by a multidisciplinary team of clinicians in Riegel et al. [3] with videos, visits, and telephone calls for 6 months and participants were followed for 6 months after discharge from hospital. As a result, length of hospital stay was approximately one day shorter for the intervention group, and acute care resource use was lower in the same group. However, effectiveness after 12 months was not demonstrated. Also, in Koelling et al. [17], nurses educated patients in one-to-one teaching session before discharge and conducted follow-up by telephone at 30, 90, and 180 days after discharge. Self-care measure scores improved at 30 days in the intervention group and the overall cost of care was lower in the same group during 180 days. On the other hand, program efficacy was not reported after 180 days. In comparison with these studies, a researcher who was a nurse consistently implemented intervention and follow-up through individual interviews with face-to-face contact from baseline up to 36 months after program commencement in our study. Accordingly, it was considered that a relationship between participants in intervention group and the researcher was established and that participants continued self-monitoring, leading to the prevention of deteriorating symptoms related to CHF throughout the follow-up period.

The results of our study showed that continuous long-term support for self-management behavior related to individual lifestyle and habits such as sodium-restricted diets and giving up smoking or drinking in particular were very important for CHF outpatients. Hence, it was necessary for nurses to give support for behavior related to individual lifestyle and habits in a busy medical environment with the aim of preventing CHF deterioration and readmission to hospital.

\subsection{Limitations of this Study and Future Challenges}

There were data collection limitations for the control group in this study. Data for the control group after 12 months was excluded because approximately $80 \%$ of the participants withdrew. Some of them were tired of the long-term research period, and others were busy and felt it was difficult for them to participate in individual interviews in every research period. At the baseline, we proposed that control group could participate in this program after the intervention group follow-up period if they wanted to. However, no one was interested. It was considered that the time frame of more than 12 months was too long for the participation of the control group. Therefore, consideration of the control group was important in order to be relieved of the burden of long term research in the future.

There were also assessment tool limitations. We used self-reported measures to assess participants' compliance with a sodium-restricted diet, medicine administration, activities/exercises, weight-monitoring and giving up smoking and drinking because there were no valid self-reporting measures in Japan. However, it was doubtful whether this was valid, so we also drew upon data from whether or not there were changes in physiological data and deteriorating symptoms related to CHF. For future research, the development of a verified assessment tool is needed for reliable data collection.

Additionally, there were limitations due to subjective assessments by the researcher. Only one researcher conducted the interventions and assessed participants' compliance in this study because it was considered that a familiar researcher gave the participants a sense of reassurance. However, there was the possibility of bias introduced by any subjective assessments conducted by the researcher, who also conducted interventions, as well as by subjects who might be motivated to please their researcher with positive accomplishments. 


\section{Conclusions}

The disease management program in Otsu and Moriyama [9] showed promise in preventing CHF outpatients from deteriorating significantly on a long-term basis because self-monitoring of activity and weight continued and there were no participants with CHF who deteriorated in the intervention group at 36 months after program commencement, although the program aimed only to provide illness and self-management knowledge. On the other hand, future work will need to compare participants in this program to a control group over an extended period of time with consideration for relieving the burden of the control group.

On the other hand, it was hard for outpatients to change their behavior concerning sodium restricted diets and quitting smoking or drinking just by providing illness and self-management knowledge because it related to personal taste and preference. Thus, it was important for outpatients to receive long-term continuous support regarding personal taste and preference.

\section{Acknowledgements}

We are extremely grateful to all participants and the collaboration of Dr. Yuzo Mori and chief nurse, Shuko Uchiumi at the Mori Heart Clinic.

\section{References}

[1] Shibata, N., Watanabe, J., Shinozaki, T., Koseki, Y., Sakuma, M., Kagaya, Y., Shirato, K., CHART Investigators (2004) Analysis of Chronic Heart Failure Registry in the Tohoku District Third Year Follow-Up. Circulation Journal, 68, 427-434. http://dx.doi.org/10.1253/circj.68.427

[2] Stewart, S., Marley, J.E. and Horowitz, J.D. (1999) Effects of a Multidisciplinary, Home-Based Intervention on Planned Readmissions and Survival among Patients with Chronic Congestive Heart Failure: A Randomized Controlled Study. Lancet, 354, 1077-1083. http://dx.doi.org/10.1016/S0140-6736(99)03428-5

[3] Riegel, B., Carlson, B., Glaser, D. and Hoagland, P. (2000) Which Patients with Heart Failure Respond Best to Multidisciplinary Disease Management? Journal of Cardiac Failure, 6, 290-299. http://dx.doi.org/10.1054/jcaf.2000.19226

[4] Smeulders, E.S., van Haastregt, J.C., van Hoef, E.F., van Eijk, J.T. and Kempen, G.I. (2006) Evaluation of a Self-Management Programme for Congestive Heart Failure Patients: Design of a Randomized Controlled Trial. BMC Health Services Research, 6, 91. http://www.biomedcentral.com/content/pdf/1472-6963-6-91.pdf http://dx.doi.org/10.1186/1472-6963-6-91

[5] Sidorov, J. (2006) Reduced Health Care Costs Associated with Disease Management for Chronic Heart Failure: A Study Using Three Methods to Examine the Financial Impact of a Heart Failure Disease Management Program among Medicare Advantage Enrollees. Journal of Cardiac Failure, 12, 594-600. http://dx.doi.org/10.1016/j.cardfail.2006.07.006

[6] Peters-Klimm, F., Müller-Tasch, T., Schellberg, D., Gensichen, J., Muth, C., Herzog, W. and Szecsenyi, J. (2007) Rationale, Design and Conduct of a Randomised Controlled Trial Evaluating a Primary Care-Based Complex Intervention to Improve the Quality of Life of Heart Failure Patients: HICMan (HeidelbergIntegrated Case Management). BMC Cardiovascular Disorders, 7. http://www.biomedcentral.com/1471-2261/7/25

[7] Smeulders, E.S., van Haastregt, J.C., Janssen-Boyne, J.J., Stoffers, H.E., van Eijk, J.T. and Kempen, G.I. (2009) Feasibility of a Group-Based Self-Management Program among Congestive Heart Failure Patients. Heart Lung, 38, 499-512. http://dx.doi.org/10.1016/j.hrtlng.2009.01.007

[8] Smeulders, E.S., van Haastregt, J.C., Ambergen, T., Uszko-Lencer, N.H., Janssen-Boyne, J.J., Gorgels, A.P., Stoffers, H.E., Lodewijks-van der Bolt, C.L., van Eijk, J.T. and Kempen, G.I. (2010) Nurse-Led Self-Management Group Programme for Patients with Congestive Heart Failure: Randomized Controlled Trial. Journal of Advanced Nursing, 66, 1487-1499. http://dx.doi.org/10.1111/j.1365-2648.2010.05318.x

[9] Otsu, H. and Moriyama, M. (2011) Effectiveness of an Educational Self-Management Program for Outpatients with Chronic Heart Failure. Japan Journal of Nursing Science, 8, 140-152. http://dx.doi.org/10.1111/j.1742-7924.2010.00166.x

[10] Lowery, J., Hopp, F., Subramanian, U., Wiitala, W., Welsh, D.E., Larkin, A., Stemmer, K., Zak, C. and Vaitkevicius, P. (2012) Evaluation of a Nurse Practitioner Disease Management Model for Chronic Heart Failure: A Multi-Site Implementation Study. Congestive Heart Failure, 18, 64-71. http://dx.doi.org/10.1111/j.1751-7133.2011.00228.x

[11] Otsu, H. and Moriyama, M. (2012) A Follow-Up Study for a Disease Management Program for Chronic Heart Failure 24 Months after Program Commencement. Japan Journal of Nursing Science, 9, 136-148.

http://dx.doi.org/10.1111/j.1742-7924.2011.00194.X 
[12] Azeyanagi, A., Oshima, M., Kamide, T., Yamahara, Y., Ebisawa, T., Umamoto, I., Nakagawa, H., Kubota, S. and Okamura, Y. (2005) The Present Situation of Elderly Patients with Heart Failure Who Were under Medical Treatment in the Hospital-An Investigation of the Current Situation and Problems among very Elderly Patients with Heart Failure. Kyoto Medical Journal, 52, 71-76.

[13] Otsu, H. and Moriyama, M. (2008) The Realities Regarding Self-Management of Chronic Heart Failure and the Relationship between Self-Management and Clinical Indicator of Chronic Heart Disease. Journal of Health Sciences Hiroshima University, 7, 66-76.

[14] Prochaska, J.O., Butterworth, S., Redding, C.A., Burden, V., Perrin, N., Leo, M., Flaherty-Robb, M. and Prochaska, J.M. (2008) Initial Efficacy of MI, TTM Tailoring and HRI's with Multiple Behaviors for Employee Health Promotion. Preventive Medicine, 46, 226-231. http://dx.doi.org/10.1016/j.ypmed.2007.11.007

[15] Otsu, H., Moriyama, M. and Nakaya, T. (2010) Development of a Japanese Version of the MacNew Heart Disease Health-Related Quality of Life Questionnaire. Journal of Japan Academy of Nursing Science, 30, 91-99. http://dx.doi.org/10.5630/jans.30.1_91

[16] Höfer, S., Lim, L.L., Guyatt, G.H. and Oldridge, N. (2004) The MacNew Heart Disease Health-Related Quality of Life Instrument: A Summary. Health and Quality of Life Outcomes, 2, 3. http://www.biomedcentral.com/content/pdf/1477-7525-2-3.pdf http://dx.doi.org/10.1186/1477-7525-2-3

[17] Koelling, T.M., Johnson, M.L., Cody, R.J. and Aaronson, K.D. (2005) Discharge Education Improve Clinical Outcomes in Patients with Chronic Heart Failure. Circulation, 111, 179-185. http://dx.doi.org/10.1161/01.CIR.0000151811.53450.B8

[18] Blue, L., Lang, E., McMurray, J.J., Davie, A.P., McDonagh, T.A., Murdoch, D.R., Petrie, M.C., Connolly, E., Norrie, J., Round, C.E., Ford, I. and Morrison, C.E. (2001) Randomised Controlled Trial of Specialist Nurse Intervention in Heart Failure. British Medical Journal, 323, 715-718. http://dx.doi.org/10.1136/bmj.323.7315.715

[19] Noordman, J., de Vet, E., van der Weijden, T. and van Dulmen, S. (2013) Motivational Interviewing within the Different Stages of Change: An Analysis of Practice Nurse-Patient Consultations Aimed at Promoting a Healthier Lifestyle. Social Science \& Medicine, 87, 60-67. http://dx.doi.org/10.1016/j.socscimed.2013.03.019

[20] Butler, C.C., Simpson, S.A., Hood, K., Cohen, D., Pickles, T., Spanou, C., McCambridge, J., Moore, L., Randell, E., Alam, M.F., Kinnersley, P., Edwards, A., Smith, C. and Rollnick, S. (2013) Training Practitioners to Deliver Opportunistic Multiple Behaviour Change Counselling in Primary Care: A Cluster Randomised Trial. British Medical Journal, 346, 10-13. http://dx.doi.org/10.1136/bmj.f1191

[21] The Japanese Dental Health Society, The Japanese Society of Oral and Maxillofacial Surgeons, The Japanese Society of Public Health, The Japanese Respiratory Society, The Japan Society of Obstetrics and Gynecology, The Japanese Circulation Society, The Japan Pediatric Society, The Japanese College of Cardiology and The Japan Lung Cancer Society (2010) Guidelines for Smoking Cessation. 1-85.

[22] The Japanese Circulation Society (2010) Guidelines for Treatment of Chronic Heart Failure. 1-85.

[23] Masunaga, N., Kimura, A., Miyataka, M. and Ichikawa, T. (2003) Effects of Cardiac Events Caused by Drinking in Patients with Old Myocardial Infarction. Medical Journal Kinki University, 28, 223-238.

[24] West, J.A., Miller, N.H., Parker, K.M., Senneca, D., Ghandour, G., Clark, M., Greenwald, G., Heller, R.S., Fowler, M.B. and DeBusk, R.F. (1997) A Comprehensive Management System for Heart Failure Improves Clinical Outcomes and Reduces Medical Resource Utilization. The American Journal of Cardiology, 79, 58-63. http://dx.doi.org/10.1016/S0002-9149(96)00676-5 